\title{
Clinical application of an intelligent oropharyngeal swab robot: implication for the COVID-19 pandemic
}

\author{
To the Editor:
}

Coronavirus disease 2019 (COVID-19), caused by infection of severe acute respiratory syndrome coronavirus 2 (SARS-CoV-2), is transmitted through respiratory droplets and close contact [1-4]. To diagnose COVID-19, oropharyngeal swab (OP swab) sampling is widely used for viral nucleic acid detection [3]. However, healthcare workers who perform OP swab are at high risk of infection due to aerosol from patients during the process of sampling. And the quality of manual OP swabs is inconsistent among different collectors, which may lead to misdiagnosis [5]. Use of a remote-controlled OP swab robot has the potential to avoid close contact between healthcare workers with patients, and thus reduce the risk of SARS-CoV-2 infection during sampling. Here, we invented a robotic sampling (RS) system and evaluated the safety and efficacy of this system on OP swab sampling during the period of pandemic.

A first-generation semi-automatic OP swab robot, jointly developed by Shenyang Institute of Automation of the Chinese Academy of Science and First Affiliated Hospital of Guangzhou Medical University, was used to take OP swabs in this study (figure 1a-d). The exposure of OP swab sampling using robot is classified according to the visible structures as follows [6]. Class I: soft palate, fauces, uvula, pillars; class II: soft palate, fauces, uvula; class III: soft palate, base of uvula; class IV: soft palate not visible at all. Class I was defined as good field of view (FOV) of posterior pharyngeal wall, and class II-IV was defined as limited FOV of posterior pharyngeal wall. Research protocol was registered (ChiCTR2000030861) and approved by the ethics committee of the First Affiliated Hospital of Guangzhou Medical University (2020-46). Suspected COVID-19 patients were determined based on the criteria of the National Health Commission of China [7].

Sampling parameters, safety and effectiveness of OP swab robot were investigated in 20 healthy individuals using four different sampling forces (10-60 g). Effectiveness was determined in terms of sampling success rate (completion in first attempt), and swab quality was determined based on the threshold cycle $(\mathrm{Ct})$ value for glyceraldehyde 3-phosphate dehydrogenase (GAPDH) by RT-PCR [8]. OP swabs with Ct values of $\leqslant 37$ and $>37$ was considered as qualified and unqualified samples, respectively. Sampling adverse reactions were determined in terms of retching, sore throat, pharyngeal congestion, or injury of posterior pharyngeal wall after sampling. Comparison study of the safety and effectiveness between RS and manual sampling (MS) was performed in 30 healthy individuals in cross-over trials. Clinical application study was performed in 20 consecutive suspected COVID-19 patients from a fever clinic to compare the pathogen test results of the two methods. OP swab sample collection in the MS group was achieved through wiping of posterior pharyngeal wall with sterile cotton swab by experienced collectors. Data normality was tested via Kolmogorov-Smirnov test and presented as mean \pm SD or median (interquartile range). Two-sided independent sample t-test, one-way ANOVA or Fisher's exact test were used as appropriate; a p-value $<0.05$ was considered statistically significant.

Sampling success rate was $95 \%$ in 20 healthy subjects with different force groups when using the RS system. The Ct values showed $97 \%$ specimens as qualified, and no significant difference in the quality of the specimens was found in the four sampling force groups based on the sample GADPH Ct value.

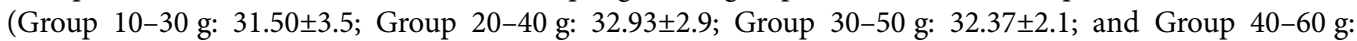

@ERSpublications

Clinical application of the safety and effectiveness of an intelligent oropharyngeal-swab robot, and its implication for the COVID-19 pandemic https://bit.ly/2BUsV55

Cite this article as: Li S-Q, Guo W-L, Liu H, et al. Clinical application of an intelligent oropharyngeal swab robot: implication for the COVID-19 pandemic. Eur Respir J 2020; 56: 2001912 [https://doi.org/ 10.1183/13993003.01912-2020]. 


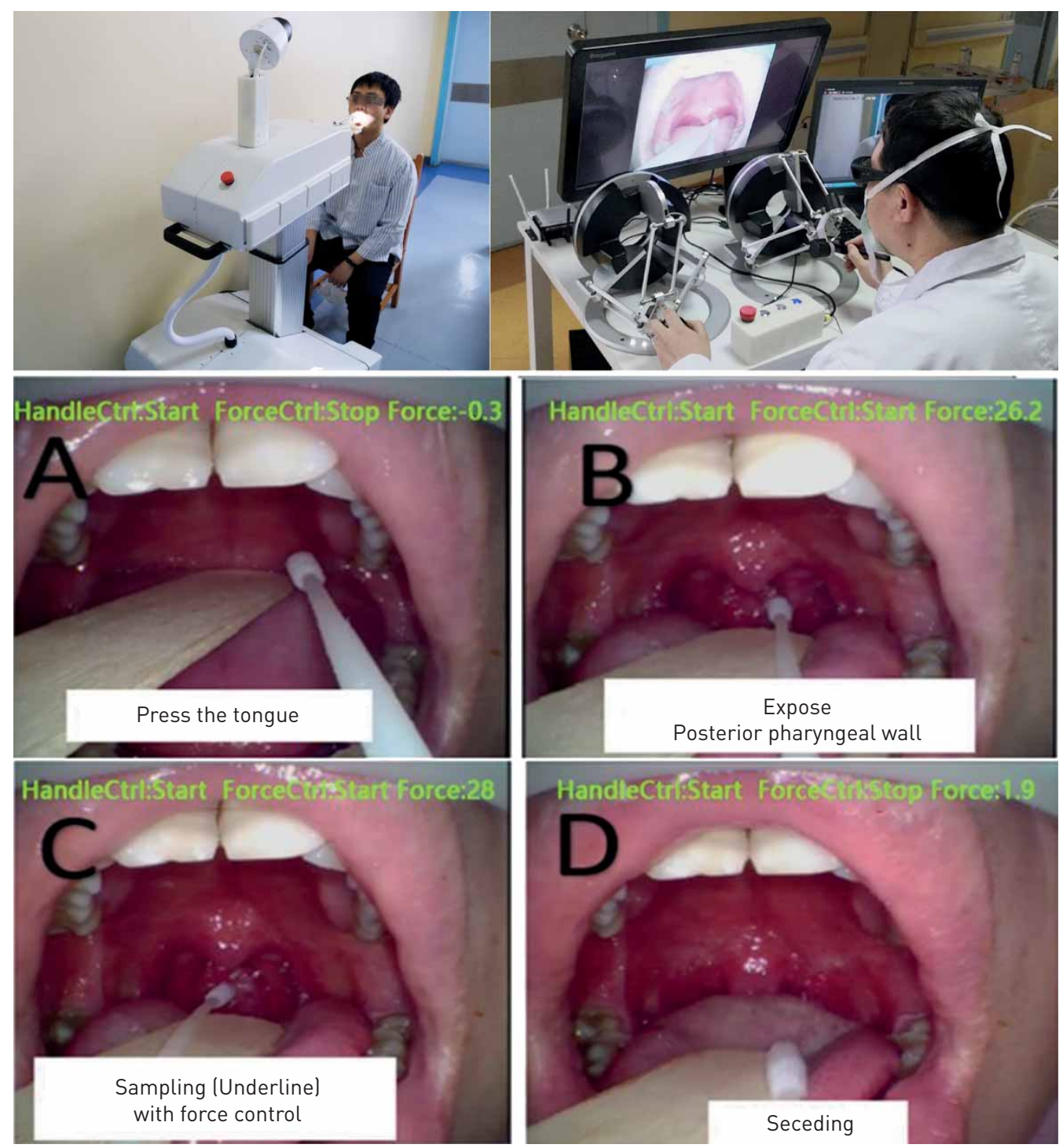

e)

\begin{tabular}{|c|c|c|c|c|c|c|}
\hline \multirow{2}{*}{$\begin{array}{l}\text { Patient } \\
\text { ID }\end{array}$} & \multicolumn{3}{|c|}{ RS species } & Shared & \multicolumn{2}{|l|}{ MS species } \\
\hline & Results & & & Pathogens & & \\
\hline 401 & + & & & Mycoplasma pneumoniae & & + \\
\hline 402 & + & & & Influenza A virus & & + \\
\hline 403 & - & & & & & - \\
\hline 404 & + & & Klebsiella pneumoniae & & & - \\
\hline 405 & - & & & & & - \\
\hline 406 & + & & & Influenza $A$ virus & & + \\
\hline 407 & + & & & Haemophilus influenzae & & + \\
\hline 408 & + & & & Mycoplasma pneumoniae & & + \\
\hline 409 & - & & & & & - \\
\hline 410 & - & & & & & - \\
\hline 411 & + & & & Influenza A virus H3N2 Klebsiella pneumoniae & & + \\
\hline 412 & + & & & Influenza A virus H1N1 & & + \\
\hline 413 & + & & Haemophilus influenzae & Influenza A virus H1N1 & & + \\
\hline 414 & + & & Influenza A virus & Haemophilus influenzae & & + \\
\hline 415 & + & & & Influenza A virus & Haemophilus influenzae & + \\
\hline 416 & + & & MRSA & Influenza A virus & Pseudomonas aeruginosa & + \\
\hline 417 & - & & & & & - \\
\hline 418 & + & & & Influenza B virus & & + \\
\hline 419 & + & Streptococcus penumoniae & Haemophilus influenzae & Mycoplasma pneumoniae & & + \\
\hline 420 & + & & Legionella pneumophila & Influenza A virus H1N1 & & + \\
\hline
\end{tabular}


FIGURE 1 Representative images of the oropharyngeal swab robot and its sampling processes. b) The force control mode was initiated, with the Force Ctrl state shown as "Start." The swab moved forward automatically until it touched the posterior pharyngeal wall and reached the pre-set force range. The current force was 26.2, as shown in the upper right corner. d) The force control mode was stopped, with the Force Ctrl state shown as "Stop." The robotic arm was controlled to withdraw the swab and retreat. e) Pathogen test results of oropharyngeal swabs collected with two different sampling methods in the 20 suspected coronavirus disease 2019 patients. RS: robotic sampling MS: manual sampling.

$32.97 \pm 2.1 ; \mathrm{p}=0.346)$. There was no pharyngeal congestion or injury in any participant; however, incidence of sore throat was higher when the sampling force is $>40 \mathrm{~g}(15.8 \%, 6 / 38)$ when compared to low-force $(\leqslant 40 \mathrm{~g})$ group $(0 / 38 ; \mathrm{p}=0.025)$. Thus, $20-40 \mathrm{~g}$ force was selected for OP swab robot sampling in subsequent experiments. Next, we compared RS and MS system and showed $95 \%$ and $100 \%$ success rates, respectively, when measuring GAPDH in the swab samples in the crossover study. All specimens were qualified based on GAPDH Ct values of $\leqslant 37$; however, variation of $\mathrm{Ct}$ value from MS specimens was more significant compared to RS specimens $(27.14 \pm 2.0$ versus $27.86 \pm 2.02 ; \mathrm{p}=0.008)$. However, the difference in $\mathrm{Ct}$ values from the data did not exceed 1, and although there was statistical difference, no clinical significance was indicated. No significant differences in adverse reactions were observed between the two groups. Swab quality was affected by limited FOV as Ct value of swab specimens with good FOV group was significantly lower than the limited FOV group (Ct value of good FOV group: 27.33 \pm 1.99 ; Ct value of limited FOV group: $28.61 \pm 1.87 ; \mathrm{p}=0.018$ ). There were seven and nine cases of limited FOV in the MS and RS groups, respectively. However, after eliminating the influence factor of limited FOV, no significant difference in swab quality was found between the MS and RS methods (27.05 \pm 2.0 versus 27.61 $\pm 1.97 ; \mathrm{p}=0.156)$. RS sampling time $(9 \pm 4.1 \mathrm{~s})$ was significantly shorter than MS group $(18 \pm 5.1 \mathrm{~s} ; \mathrm{p}=0.032)$. There was no significant difference in adverse reactions between the RS and MS groups (10\% versus $10 \%$; $\mathrm{p}=1)$.

Lastly, OP swabs were collected using MS and RS in 20 suspected COVID-19 patients, including eight males and 12 females with the mean age of $45 \pm 15$ years. OP swab samples were subjected to pathogen testing. SARS-CoV-2 nucleic acid was negative for all suspected cases. The results of viral and mycoplasma tests were negative. There were two cases tested positive for bacteria, including one case of Staphylococcus infection and another case of Moraxella catarrhalis infection.

The consistency of the pathogen discovery results between the two sampling methods for the 20 suspected cases of COVID-19 reached 95\% (Kappa=0.875). No differences in the success rate, swab quality, and pathogen discovery results were found between the OP swab robot and manual sampling methods.

Standard sampling of OP swab, including the precise delivery of swab to target tissue, appropriate force and touch avoidance of surrounding tissue, has confirmed to be prerequisite for the enhancement of positive detection rate [9-11]. MS swab sampling may potentially lead to false-negative results due to lack of experience and psychological anxiety of sampling personnel due to the contagious nature of SARS-CoV-2 [5]. OP swab robot with remote camera can help medical staffs to have clear vision during sampling without close contact to patients, thus resulting in collection of high-quality swab specimens consistently. Though greater force is believed to be capable of collecting higher number of cells per swab, our results showed that there was no correlation between the quality of swab specimens and sampling force. Thus, the RS system showed an advantage in the quality control of OP swab sampling, and have the potential to minimise the risk of infection to healthcare workers [11].

Time from research and development to application was pressed in light of the crisis of the emerging infectious disease. Therefore, the first generation of pharyngeal swab robots still required medical assistants to manually replace the isolation sleeves and swabs after collecting the specimens, which would minimise but not avoid the exposure to SARS-COV-2. However, ultraviolet disinfection and air disinfectant machine were performed for sterilisation after each specimen collection to avoid the exposure risk to the utmost extent. Additionally, in view of this situation, a second-generation intelligent robot has been developed to achieve intelligent movement and automatically replace the swab and protective cover, and is currently preparing for the next phase of clinical trials. It can further improve intelligence of the process and reduce the degree of manual participation.

Though this was a preliminary, single-centre study in limited COVID-19 patients, the sampling process of the intelligent OP swab robot is safe, with a high success rate of sampling. The quality of OP swabs and the detection rate of pathogens were not inferior to those collected manually. The OP swab robot presents a safe and effective sampling method, and could avoid cross infection to operators. During epidemics or pandemics, the OP swab robot has good prospects for application in clinical practice. 
Shao-Qiang $\mathrm{Li}^{1,3}$, Wen-Liang Guo $\oplus^{1,3}$, Hao Liu ${ }^{2,3}$, Tao Wang ${ }^{1}$, Yuan-Yuan Zhou ${ }^{2}$, Tao $\mathrm{Yu}^{2}$, Chong-Yang Wang ${ }^{2}$, Yong-Ming Yang ${ }^{2}$, Nan-Shan Zhong ${ }^{1}$, Nuo-Fu Zhang ${ }^{1,4}$ and Shi-Yue $\mathrm{Li}^{1,4}$

${ }^{1}$ State Key Laboratory of Respiratory Diseases, National Clinical Research Center for Respiratory Disease, Guangzhou Institute of Respiratory Health, The First Affiliated Hospital of Guangzhou Medical University, Guangzhou, China. ${ }^{2}$ State Key Laboratory of Robotics, Shenyang Institute of Automation, Institutes for Robotics and Intelligent Manufacturing, Chinese Academy of Sciences, Liaoning Key Laboratory of Minimally Invasive Surgical Robot, Shenyang, China. ${ }^{3}$ These authors contributed equally to this manuscript. ${ }^{4}$ Nuo-Fu Zhang or Shi-Yue Li contributed equally to this article as lead authors and supervised the work.

Correspondence: Shi-Yue Li, State Key Laboratory of Respiratory Diseases, National Clinical Research Center for Respiratory Disease, Guangzhou Institute of Respiratory Health, The First Affiliated Hospital of Guangzhou Medical University, Guangzhou, China. E-mail: lishiyue@188.com

Received: 12 May 2020 | Accepted after revision: 22 June 2020

Published in volume 56, issue 2 of the European Respiratory Journal on 20 Aug 2020; republished 30 March 2021 with amendments to specific grant number details.

Conflict of interest: S-Q. Li reports grants from National Natural Science Foundation of China (grant number 62041302), Innovative and Strategic Program of Guangdong's Scientific and Technological Policy (grant number 2020B111126005) and Guangzhou Science and Technology Innovation Development Special Fund (grant number 2060901), during the conduct of the study. W-L. Guo reports grants from National Natural Science Foundation of China (grant number 62041302), Innovative and Strategic Program of Guangdong's Scientific and Technological Policy (grant number 2020B111126005) and Guangzhou Science and Technology Innovation Development Special Fund (grant number 2060901), during the conduct of the study. H. Liu reports grants from National Natural Science Foundation of China (grant number 62041302), Innovative and Strategic Program of Guangdong's Scientific and Technological Policy (grant number 2020B111126005) and Guangzhou Science and Technology Innovation Development Special Fund (grant number 2060901), during the conduct of the study. T. Wang reports grants from National Natural Science Foundation of China (grant number 62041302), Innovative and Strategic Program of Guangdong's Scientific and Technological Policy (grant number 2020B111126005) and Guangzhou Science and Technology Innovation Development Special Fund (grant number 2060901), during the conduct of the study. Y-Y. Zhou reports grants from National Natural Science Foundation of China (grant number 62041302), Innovative and Strategic Program of Guangdong's Scientific and Technological Policy (grant number 2020B111126005) and Guangzhou Science and Technology Innovation Development Special Fund (grant number 2060901), during the conduct of the study. T. Yu reports grants from National Natural Science Foundation of China (grant number 62041302), Innovative and Strategic Program of Guangdong's Scientific and Technological Policy (grant number 2020B111126005) and Guangzhou Science and Technology Innovation Development Special Fund (grant number 2060901), during the conduct of the study. C-Y. Wang reports grants from National Natural Science Foundation of China (grant number 62041302), Innovative and Strategic Program of Guangdong's Scientific and Technological Policy (grant number 2020B111126005) and Guangzhou Science and Technology Innovation Development Special Fund (grant number 2060901), during the conduct of the study. Y-M. Yang reports grants from National Natural Science Foundation of China (grant number 62041302), Innovative and Strategic Program of Guangdong's Scientific and Technological Policy (grant number 2020B111126005) and Guangzhou Science and Technology Innovation Development Special Fund (grant number 2060901), during the conduct of the study. N-S. Zhong reports grants from National Natural Science Foundation of China (grant number 62041302), Innovative and Strategic Program of Guangdong's Scientific and Technological Policy (grant number 2020B111126005) and Guangzhou Science and Technology Innovation Development Special Fund (grant number 2060901), during the conduct of the study. N-F. Zhang reports grants from National Natural Science Foundation of China (grant number 62041302), Innovative and Strategic Program of Guangdong's Scientific and Technological Policy (grant number 2020B111126005) and Guangzhou Science and Technology Innovation Development Special Fund (grant number 2060901), during the conduct of the study. S-Y. Li reports grants from National Natural Science Foundation of China (grant number 62041302), Innovative and Strategic Program of Guangdong's Scientific and Technological Policy (grant number 2020B111126005) and Guangzhou Science and Technology Innovation Development Special Fund (grant number 2060901), during the conduct of the study.

Support statement: This study was supported by the National Natural Science Foundation of China (grant number 62041302 and 81970046), Innovative and Strategic Program of Guangdong's Scientific and Technological Policy (grant number 2020B111126005) and Guangzhou Science and Technology Innovation Development Special Fund (grant number 2060901). Funding information for this article has been deposited with the Crossref Funder Registry.

\section{References}

1 Chan JFW, Yuan S, Kok KH, et al. A familial cluster of pneumonia associated with the 2019 novel coronavirus indicating person-to-person transmission: a study of a family cluster. Lancet 2020; 395: 514-523.

2 Huang C, Wang Y, Li X, et al. Clinical features of patients infected with 2019 novel coronavirus in Wuhan, China. Lancet 2020; 395: 497-506.

3 Chen N, Zhou M, Dong X, et al. Epidemiological and clinical characteristics of 99 cases of 2019 novel coronavirus pneumonia in Wuhan, China: a descriptive study. Lancet 2020; 395: 507-513.

4 Chinese Academy of Sciences. Wuhan Coronavirus Has Strong Ability to Infect Humans. https://view.inews.qq. com/w2/20200121A0M08X00?tbkt=F\&strategy=\&openid=o04IBALMrLyGDxbWNOPoDM1IfG-s\&uid=\&refer=wX_ hot Date last accessed: 21 January, 2020.

5 Li D, Wang D, Dong J, et al. False-negative results of real-time reverse-transcriptase polymerase chain reaction for severe acute respiratory syndrome coronavirus 2: role of deep-learning-based CT diagnosis and insights from two cases. Korean J Radiol 2020; 21: 50.

6 Samsoon GL, Young JR. Difficult tracheal intubation: a retrospective study. Anaesthesia 1987; 42: 487-490. 
7 National Health Commission of the People's Republic of China. Chinese Management Guideline for COVID-19 (Version 7.0). http://www.nhc.gov.cn/xcs/zhengcwj/202003/46c9294a7dfe4cef80dc7f5912eb1989/files/ce3e6945832a 438eaae415350a8ce964.pdf

8 Radonic A, Thulke S, Mackay IM, et al. Guideline to reference gene selection for quantitative realtime PCR. Biochem Biophys Res Commun 2004; 313: 856-862.

9 Hosokawa-Muto J, Sakai H, Sassa Y, et al. Rapid detection of pathogenic virus genome sequence from throat and nasal swab samples using an exhaustive gene amplification method. Forensic Sci Med Pathol 2019; 15: 399-403.

10 van der Veen EL, Sanders EA, Videler WJ, et al. Optimal site for throat culture: tonsillar surface versus posterior pharyngeal wall. Eur Arch Otorhinolaryngol 2006; 263: 750-753.

11 Li CQL, Li YY, Wang YF, et al. Comparison among nasopharyngeal swab, nasal wash, and oropharyngeal swab for respiratory virus detection in adults with acute pharyngitis. BMC Infect Dis 2013; 13: 281. 